\title{
Revestimientos y acabados superficiales en construcciones con tierra contemporáneas
}

\author{
Rendering and finishing on contemporary earth building
}

\author{
F. I. Castilla ${ }^{(*)}$
}

RESUMEN

La creciente demanda para la construcción de muros de tierra en algunos ámbitos del sector de la edificación conlleva la necesidad de plantear soluciones constructivas que permitan el adecuado mantenimiento de las prestaciones de dichos muros. Al igual que las propias técnicas para la construcción de los muros, los materiales utilizados para su revestimiento y protección han evolucionado permitiendo una gran variedad de soluciones formales acordes a cada situación.

En este artículo se pretende hacer una revisión sobre las particularidades de las diferentes posibilidades que se presentan en el panorama de la construcción actual: desde los revestimientos que permitan mantener a la vista los acabados de los muros de tierra a los revocos de distinta naturaleza, con especial incidencia en aquellos que utilizan la propia tierra como material base, siendo los más apropiados a priori por su natural compatibilidad con el soporte. La explicación se acompañará de ejemplos ilustrativos de la aplicación de dichas técnicas en edificios construidos durante las dos últimas décadas.

113.108

Palabras clave: revestimiento, muro, tierra, construcción, barro.
SUMMARY

Earth construction is in increasing demand in some areas of building activity, which means a need for technical solutions to maintain and preserve the properties of earth walls. Like earth wall construction techniques themselves, materials used for rendering (plastering, painting, panelling, etc) have evolved in a way that allows us to find formal solutions suitable for every architectural situation.

This paper deals with the special features of each rendering technique available in contemporary construction, from renderings permitting earth wall textures and finish to be appreciated, to plasters from different materials. Special attention will be focused on clay plasters, which are more appreciated due to its natural characteristics and compatibility with the base wall.

The explanations are supported by different examples of buildings where these techniques have been used during the last two decades.

Key Words: render, wall, earth, construction, clay (mud). 


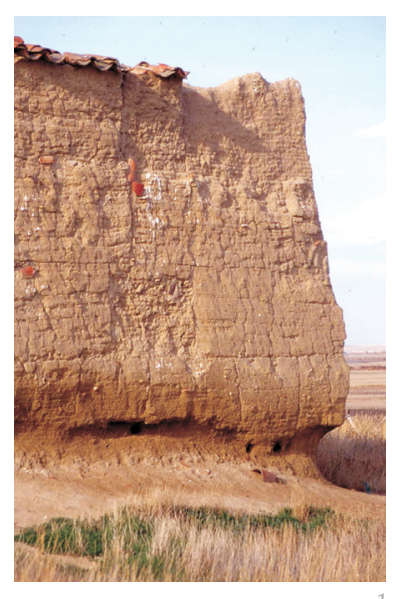

\section{INTRODUCCIÓN}

Tradicionalmente, las fábricas de tierra expuestas a la intemperie debían estar protegidas por elementos que evitasen o disminuyesen el contacto directo con el agua en aras de su durabilidad, esto es:

- Zócalos (puntidos, basamentos, sobrecimientos) de materiales más resistentes y sobre todo menos permeables (de menor coeficiente de absorción) que eviten o disminuyan la ascensión capilar del agua procedente del terreno y las posibles salpicaduras

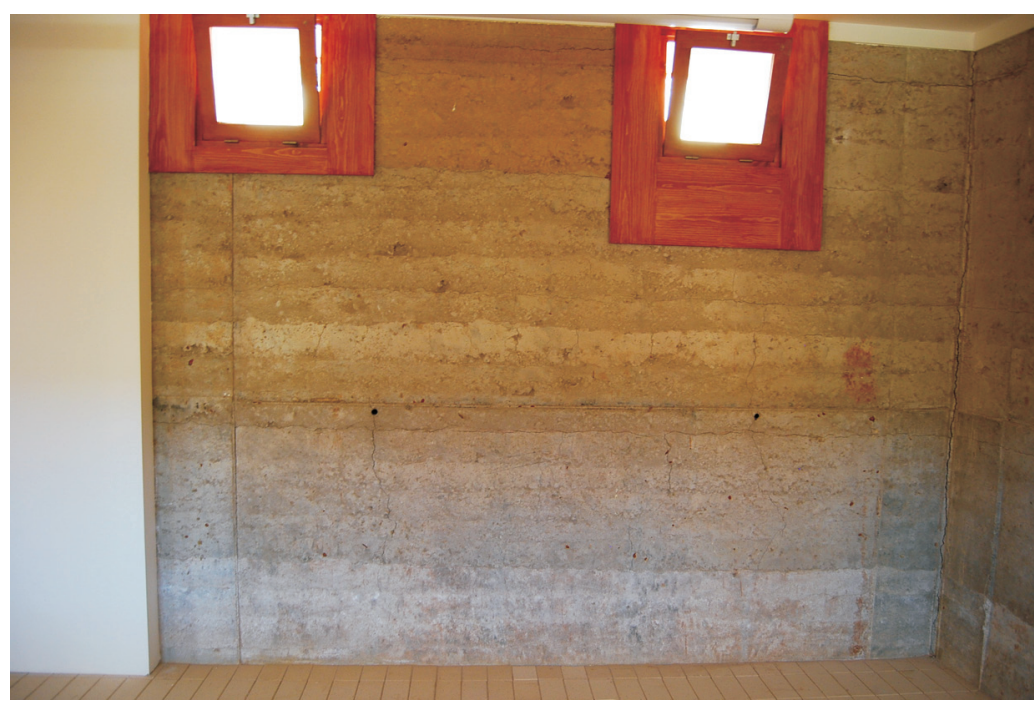

1. Proceso de degradación de muro de tierra sin protección. Santa María de los Oteros (Palencia)

2. Muro de tierra apisonada de 70 $\mathrm{cm}$ de espesor, aula de Naturaleza en Pozuelo de Alarcón (Madrid). Arquitecto: Antonio Baño. Enero 2008

1 En muchas ocasiones, especialmente cuando los muros superan estas dimensiones, lo habitual es que estos se carearan con mampostería, sillares o simple calicostrado (ver apartado 3 de este artículo) siendo la tierra material de relleno interior.
- Aleros, cornisas o remates de coronación del muro que eviten la agresión directa del agua de lluvia y el correspondiente escurrimiento y erosión provocados por la misma.

- Protección de la superficie del paramento por alguno de los procedimientos habituales de aplacado, revestimiento continuo con morteros de distinta índole (revocos), pinturas o impregnaciones superficiales.

Tan sólo en la construcción militar o monumental de muros de grandes secciones (fortificaciones, castillos, recintos amurallados) de tapia de tierra, donde se pueden alcanzar espesores de $90 \mathrm{~cm}$ a $2 \mathrm{~m}$, los paramentos carecían de alguno de estos elementos o no se revestían, si bien en la mayoría de los casos se trataba de tierra mejorada con algún conglomerante, más cercana a un hormigón pobre, a pesar de estar apisonada ${ }^{1}$.

En muchos casos no era necesario "mejorar" la propia tierra del lugar para la construcción de los muros; bastaba con proteger el muro con los elementos mencionados. La aparente debilidad de las construcciones de tierra se debe a la frecuente inexistencia o desaparición de estos elementos a lo largo de la vida de los edificios, quedando las fábricas expuestas a la acción directa del agua. Cuando la construcción se ha abandonado a su suerte, la rotura de una simple teja sobre un muro es el desencadenante habitual del proceso que lleva a su ruina casi completa. Las huellas de este proceso se pueden apreciar en la mayoría de muros, que a pesar de su elevado grado de erosión (Figura 1) mantienen su estabilidad.

En la construcción popular es habitual encontrar los muros de tierra, especialmente en fachadas, con algún tipo de protección superficial, mediante alguno de los procedimientos mencionados, que se renovaban con mayor o menor periodicidad, en función de la disponibilidad de materiales y recursos. Incluso en el interior de las construcciones, por razones de higiene, o para evitar el desprendimiento de partículas del muro, se realizaba, siempre que era posible, algún tipo de protección superficial. No obstante, esto siempre depende de las exigencias de bienestar e higiene y está asociado al nivel de vida de sus ocupantes, por lo que en zonas de escasos recursos (incluso en la actualidad) no es extraño encontrar algún que otro ejemplo en que adobes, tapias, rellenos de barro, etc, aun tratándose de edificaciones de uso residencial, no presentan revestimiento alguno.

Las técnicas de construcción y materiales desarrollados en el último siglo permiten la utilización de la tierra en una amplia variedad de soluciones constructivas que, si bien tienen su origen en la construcción tradicional, se alejan de ésta en cuanto a sus criterios de utilización y prestaciones. En la arquitectura contemporánea de nuestro entorno próximo, y en el marco normativo de la edificación en la UE, las exigencias de confort ambiental y salubridad nos obligan en la mayoría de las situaciones a la utilización de la tierra mejorada para la construcción de cerramientos que queden expuestos a la intemperie o a la utilización de soluciones de revestimiento para su protección. Estos revestimientos deben cumplir una doble finalidad: garantizar la durabilidad del material que compone el muro de cerramiento y en consecuencia la integridad (y estabilidad) del elemento constructivo y garantizar el comportamiento higrotérmico previsto de dicho cerramiento (no viéndose afectado éste por la presencia de humedad).

Otro de los aspectos que ha tomado especial relevancia en la actualidad son las cualidades plásticas de los paramentos de tierra, por lo que la decisión relativa a su revestimiento, y en consecuencia a mostrar o no su apariencia, responde en muchas ocasiones a cuestiones ajenas a la idoneidad "técnica" de la solución constructiva, debiendo cumplir en cualquier caso con las exigencias mencionadas. La necesidad e idoneidad del revesti- 
miento vendrá dada por tanto, en cada caso, por la ubicación del paramento -interior o exterior- y su grado de exposición.

\section{MUROS DE TIERRA SIN REVESTIR}

Siempre que las condiciones climáticas lo permitan ${ }^{2}$, en el caso de cerramientos exteriores, hoy en día es posible encontrar soluciones constructivas que mejoran el comportamiento de la tierra a la intemperie con suficientes garantías como para dejar el material expuesto. Es el caso de la tierra apisonada (generalmente estabilizada) o los BTC (Figuras 2 y 3). Si el muro está compuesto por elementos de mayor capacidad de absorción de agua, como los adobes o tierras sin estabilizar, esto también es posible siempre que se encuentre protegido por porches o galerías amplias. En caso de conformarse el cerramiento mediante una única hoja, el muro debe tener el espesor suficiente $(40 \mathrm{~cm}$ o más) como para garantizar un comportamiento higrotérmico adecuado si no se va a aplicar ningún tipo de tratamiento superficial.

En el caso de particiones interiores, o cuando la fábrica (generalmente de BTC) conforma la hoja interior del muro de cerramiento, la opción de la tierra "vista" es más frecuente dado que las exigencias son menores.

\section{MUROS REVESTIDOS}

Por revestimiento entenderemos cualquier tratamiento o elemento aplicado durante o después de la construcción del muro con la finalidad de mejorar alguna de sus propiedades o con intención de aportar un determinado aspecto a la superficie del paramento. En el primer caso debemos preocuparnos de que el revestimiento cumpla con su finalidad y en ambos de que dicho revestimiento sea compatible y no perjudique ninguna otra característica del muro. Atendiendo a la naturaleza del material utilizado y su forma de aplicación, distinguimos las siguientes soluciones, cuyas particularidades, en el caso de los muros de tierra, son el objetivo principal de este artículo:

\subsection{Consolidación de la superficie. Imprimaciones e impregnaciones}

Uno de los métodos para conseguir una protección adicional, endurecimiento y/o mayor resistencia frente a la erosión, dejando a la vista la apariencia de la fábrica de tierra, consiste en el tratamiento superficial del muro a posteriori mediante impregnación (aplicado con rodillo o mediante pulverización) o lechada (mediante brocha o rodillo) de productos naturales o sintéticos, de aspecto más o menos transparente.
Son numerosos los ejemplos y las publicaciones $^{3}$ que documentan la utilización de este tipo de materiales, que por su variedad y dificultad de identificación (en muchos casos el nombre comercial sustituye al compuesto o al material de que se trata) hacen difícil compararlos entre sí y extraer conclusiones generalizables. Se resumen a continuación algunas experiencias concretas y sus resultados.

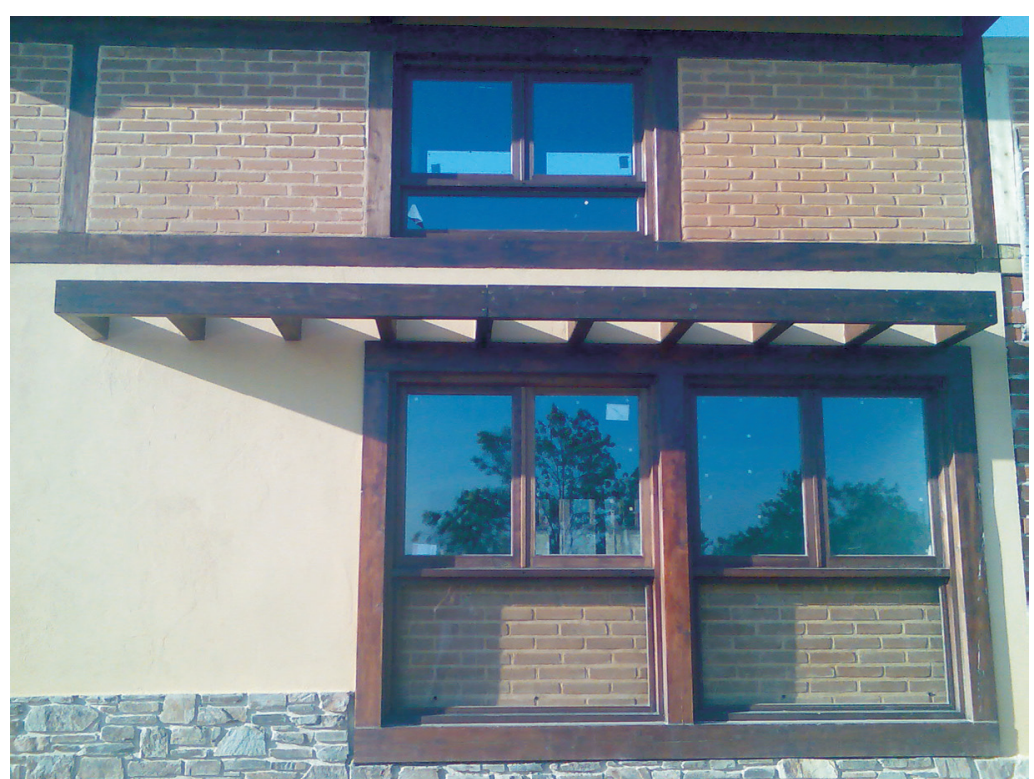

\subsubsection{Impregnaciones superficiales}

Son muchos los estudios que han pretendido determinar el comportamiento de productos consolidantes (entendiendo como tales, productos aplicados superficialmente) desde hace décadas. Destacan por su exhaustividad los realizados en las ruinas arqueológicas de Fort Selden (Nuevo México) y Mesa Verde (Arizona). En el primero, realizado a finales de los años ochenta, se aplicaron distintos tratamientos directamente sobre los muros y sobre revestimientos de barro previos $(1,2)$. Los resultados fueron mejores en los morteros estabilizados que en las imprimaciones superficiales, y dentro de los productos utilizados para los primeros se mencionan las emulsiones de resinas acrílicas como las que mejor se comportan ${ }^{4}$. En el caso de Mesa Verde, (3) el tratamiento no se aplicó sobre las fábricas, sino sobre cinco tipos de revocos utilizados tradicionalmente para el revestimiento de dichas fábricas de tierra: tierra, cal, yeso, cal/ tierra y cal/yeso (aunque no se especifican las dosificaciones).

Los consolidantes estudiados fueron:

Mucílago de tuna (cactus de la familia Opuntia Ficus indica), usado tradicionalmente en numerosas zonas de Sudamérica como consolidante y/o aditivo. Reduce la aparición de bacterias.

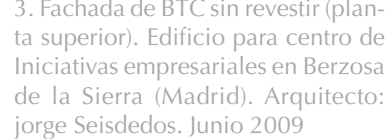
jorge Seisdedos. Junio 2009 
4. Pintura de Cal sobre paramento interior de cerramiento de adobe estabilizado. Vivienda unifamiliar. (Taos, Nuevo México, EEUU) Agosto 1998

5. Muro interior de BTC con pintura de caseina. Vivienda Unifamiliar. Berlín (Alemania)
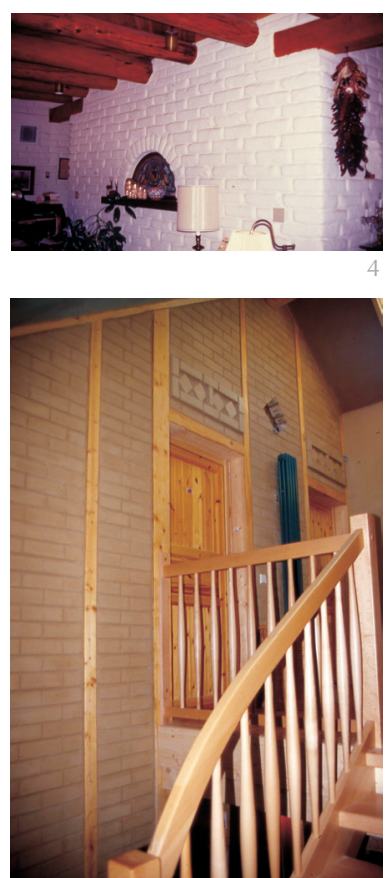

${ }^{5}$ Sin especificar (sólo se indica que se realizó durante 28 días). ${ }^{6}$ Fuente: www.construtierra.org/ construtierra_ejemplos.html

${ }^{7}$ Fuente: Antonio Raya de Blas. Arquitecto, autor del proyecto.

${ }^{8}$ Fuente: Gabriel Barbeta. Doctor Arquitecto, autor del proyecto.
Acryloid B-67 (Rohm \& Hass): resina/emulsión acrílica de metacrilato de Isobutilo. Se aplica diluido en dietilo de benceno (al 5\%) para permitir una completa impregnación. (Aunque se conocían algunas desventajas: poca penetración y cambios de color y brillo en la superficie del revestimiento).

Conservare $\mathrm{OH}$ - Stone strengthener (ProSoCo): silicato de etilo y metiletilcetona y acetona.

De las combinaciones realizadas entre los cinco revestimientos mencionados y los consolidantes aplicados a los mismos, el sistema soporte-revestimiento que demostró mayor compatibilidad (a pesar de su baja resistencia a la erosión hídrica), sin aplicar consolidantes, fue el de revoco de tierra (como por otro lado parece lógico) y el peor el de cal/ yeso. Los ensayos se realizaron aplicando revestimientos de unos $3 \mathrm{~mm}$ de espesor sobre probetas construidas con material procedente de adobes antiguos y estos fueron: Abrasión, por chorro abrasivo; Adhesión, mediante probetas en forma de ocho; $\mathrm{Ab}$ sorción (capilaridad) por cantidad absorbida colocando tubos graduados; Resistencia al agua, mediante goteo durante dos horas y Transmisión de vapor ${ }^{5}$.

En la mayoría de los casos el tratamiento mejoró el comportamiento de los revocos y el mucílago fue el que lo hizo en menor grado. En los revestimientos tradicionales de tierra, B-67 produjo una reducción intensa de absorción, pero también produjo fisuración y Conservare $\mathrm{OH}$ (silicato de etilo), fue el que más disminuyó la difusividad.

Precisamente sobre este producto (silicato de etilo) encontramos numerosas referencias en otros estudios ya que desde hace treinta años se viene utilizando, no sin cierta controversia, y parece muy efectivo en la consolidación de construcciones de tierra (1). Es un compuesto en parte orgánico, pero tras el curado la sustancia depositada es completamente inorgánica. El producto reacciona generando fuertes enlaces químicos entre las partículas de arcilla, evitando la separación de las láminas de arcilla en presencia de agua. Se produce un aumento de la resistencia a la erosión del agua, sin ser hidrófugo y permitiendo el paso del vapor por microporos. Por otro lado, su aplicación es irreversible y no funciona bien sobre superficies húmedas.

Esta misma referencia encontramos en la restauración de una casa de campo "AlUdhaibat" en Arabia Saudí (4) con adobe y embarrado. En este trabajo se menciona que con el tiempo se ha demostrado que la única sustancia que funciona sobre el barro es el silicato de etilo. Como ventajas menciona la capacidad de transpiración del muro (mantiene la permeabilidad a la vez que repele el agua) y su capacidad de impregnación sin formar una película. El producto recomendado era Ethyl Silicate 40 (silester ZLS) diluido en alcohol etílico. El tratamiento se tiene que repetir periódicamente ya que las Iluvias torrenciales han dañado los revestimientos en las zonas más expuestas a pesar del tratamiento.

Como referencia más reciente a la alternativa de productos de origen natural, un estudio realizado a propósito de la intervención en el conjunto arqueológico de Teotihuacán en México (5) plantea la utilización de sustancias procedentes de jugos de plantas, residuos de cocción de jugo de caña de azúcar y semillas hervidas en agua similares como consolidantes de los adobes por impregnación superficial con buenos resultados.

Estas investigaciones, generalmente derivadas de las necesidades del sector de la restauración e intervención en el patrimonio, han trascendido dicho ámbito, aplicándose igualmente a muros de nueva construcción, con la intención de permitir (voluntaria u obligadamente) la percepción visual del muro de tierra.

En ejemplos más recientes de la Península Ibérica podemos encontrar algunas alternativas que se han probado con éxito, a falta de que el paso del tiempo sancione la bondad de las mismas:

- Consolidación de revestimiento de barro sobre fábrica de adobe con Durcisseur de Mur POUT PRE, en casa rural de Villalón de Campos (Valladolid) ${ }^{6}$

- Consolidación de muros de tierra apisonada estabilizada en la piscina climatizada municipal de Toro (Zamora) con SIKAGUARD $710^{7}$ consistente en la protección de la superficie mediante siloxanos en base orgánica, mediante inundación en dos manos, con efecto algicida, fungicida e hidrofugante con efecto perlante.

- Consolidación de paramentos interiores de BTC en la Escuela Infantil de Bressol (Gerona), mediante aplicación de silicato potásico diluido con agua con tierra del lugar ${ }^{8}$.

- Consolidación superficial de paramentos interiores de tierra apisonada en Aula de Naturaleza en Pozuelo de Alarcón (Madrid) con CONIMACRIL RDD (Qimivisa S.L.).

Cuando se trata de garantizar una resistencia superficial y evitar exclusivamente el 
desprendimiento de partículas ocasionado por la erosión mecánica (producida por el uso cotidiano de los espacios habitables) en paramentos interiores, la gama de productos resulta más amplia, presentando menos dificultades. El silicato potásico es uno de los productos más recurrentes. Los productos naturales comercializables (caseína, linaza, etc.) son también habituales, primando para su utilización los criterios de repercusión medioambiental frente a otros productos (6). Estos productos son también utilizados con frecuencia como estabilizantes de lechadas y pinturas, como veremos a continuación.

\subsubsection{Pinturas y lechadas estabilizadas}

Las pinturas pueden estar formadas por una lechada de la propia tierra, mezclada con otros productos, o por materiales completamente distintos a la tierra.

La pintura o lechada de cal es otra de las soluciones que ha caracterizado la imagen de la arquitectura popular en numerosas partes de la geografía española y particularmente en la mitad sur de la península. Las bondades de los encalados y su compatibilidad con los muros de tierra han sido sobradamente tratadas por la bibliografía sobre arquitectura popular. Aplicados sobre cualquier soporte, a veces como acabado de una primera capa de revoco con mortero de otro material y a veces aplicados sobre la superficie irregular del propio muro, proporcionan un ligero velo que enfatiza las propias irregularidades y rugosidades del mismo, creando un juego de luces y sombras donde no caben las superficies planas ni lisas (Figuras 4 y 5).

Las pinturas para exteriores deben de ser impermeables al agua, pero a la vez deben ser porosas, con una red continua de micro capilares que permita la difusión del vapor hacia el exterior (especialmente en climas fríos). En este sentido las combinaciones más habituales en la actualidad son las de cal, con borax y/o caseína (6) y las pinturas al silicato, de aplicación habitual sobre soportes de tipo pétreo.

Además de los productos mencionados (quizás de más actualidad por la disponibilidad comercial de los mismos en droguerías industriales) la utilización de otros compuestos como sebo o grasa animal, orina, clara de huevo, engrudos (de harina de centeno) y jugos o aceites vegetales (principalmente agave, cactus, palma, kapok) aparece continuamente citada en las fuentes de cultura popular y procedimientos tradicionales, siendo aplicados localmente en numerosas experiencias de autoconstrucción y/o vivienda de bajo coste, con recetas muy variadas.
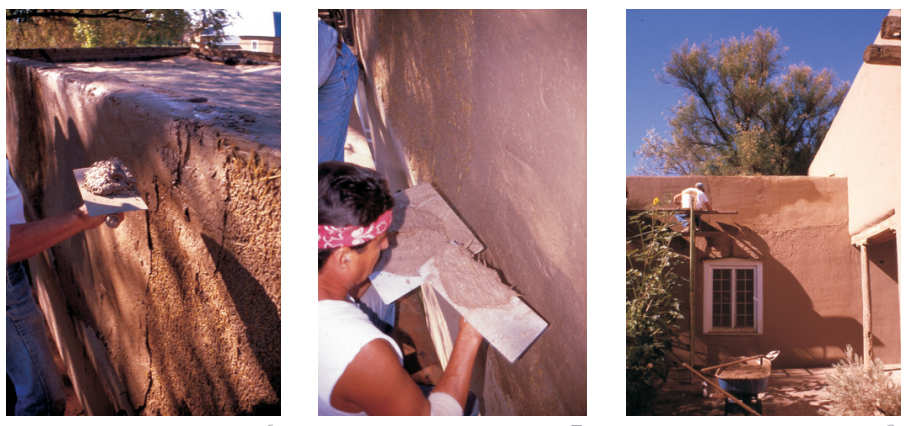

\subsection{Revocos}

Si las pinturas en general son revestimientos aplicados con brocha, rodillo o pulverizados, por revocos entenderemos aquellos revestimientos continuos conformados a partir de una pasta o mortero con consistencia plástica aplicados con la mano, paleta, Ilana o proyectados.

Uno de los principales requisitos del revestimiento consiste en lograr una máxima adherencia entre el mismo y el soporte a través de una similitud de rigideces. Por su parte, el soporte deberá ser suficientemente sólido para no desprenderse con el peso del revestimiento.

\subsubsection{Revestimientos exteriores}

La solución más adecuada, en términos generales, es la utilización de la propia tierra a modo de embarrado (trullado). Dado que en su fabricación tradicional suele producirse agrietamiento, es necesario utilizar fibras (paja tradicionalmente) que minimicen la fisuración producida por la retracción durante el secado. Sobre las proporciones existen testimonios muy variados, desde dos partes de paja por cada una de barro al inverso, La falta de conocimiento previo y la pérdida de la costumbre no permiten establecer con anterioridad al amasado las proporciones ideales en cada caso. Por otro lado estas proporciones "ideales" resultan de un acuerdo entre la mínima cantidad necesaria para alcanzar el nivel de fisuración que se considere aceptable y la suficiente adherencia o pegajosidad del embarrado, lo que una vez más obliga a la realización de una serie de muestras previas. El método más aconsejable en cualquier caso es la observación y determinación de esta proporción en los edificios de construcción tradicional más próximos.

Si el espesor del revestimiento debe superar los $15 \mathrm{~mm}$ se tiene que aplicar en dos capas; la capa de base debe contener más arcilla y áridos gruesos que la de acabado. El hecho de que la capa de base presente fisuraciones no es problemático, sino que incluso puede proporcionar una mejor ad-

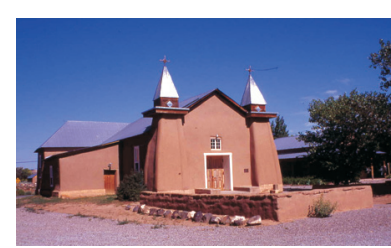

6-8. Revestimiento de barro en la rehabilitación de Casa Museo en Yan Ysidro (NM, EEUU) Julio 1998. San Ysidro (NM, EEUU) Julio 1998. El procedimiento consiste en la aplicación de de una imprimación superficial de producto hidrofugante diluido con agua sobre el muro de adobes. Una primera capa de mortero (barro y paja) amasada con misma disotución con que se a misma disolución con que se realiza la imprimación (para mejorar la adherencia) y una capa final de acabado más fina estabilizada con emulsión asfáltica y sin paja 


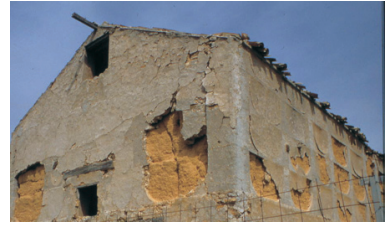

10

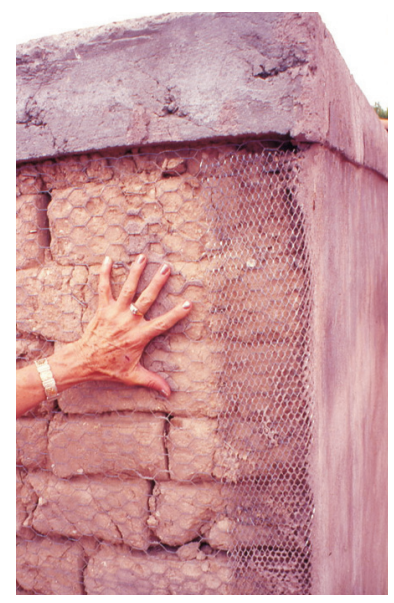

11

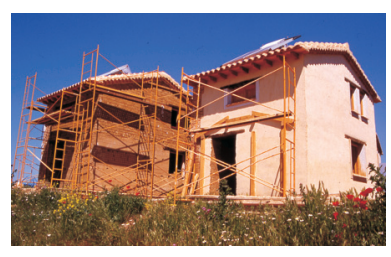

12

10. Revestimiento de enfoscado de cemento sobre muro de tapia de tierra con brencas. Tembleque (Toledo)

11. Aplicación de revoco de cemento coloreado sobre muro de adobe con colocación de tela de gallinero sobre el paramento. Agosto 2000

12. Revoco de cal en viviendas unifamiliares de muros de adobe y tapia de $50 \mathrm{~cm}$ de espesor, en Amayuelas (Palencia). Se ha utilizado (tanto interior como exterior) un enfoscado de cal, arena (1:4) y paja (un puñado por palada de arena) y sobre éste a las $24 \mathrm{~h}$ y tras humedecerlo de nuevo un acabado igual pero en proporciones 1:2 (con la misma cantidad de paja), según testimonios directos de los constructores

13. Gunitado sobre muro exterior de BTC en edificio para Escuela Bresol (Gerona) El mortero proyectado está compuesto por una parte cal aérea, media de cemento blanco, dos de arena y dos de tierra del lugar. (Foto Gabriel Barbeta) herencia a la capa fina del revestimiento. En muchos casos sirven de base a otro tipo de revocos o para un enlucido final con cal o con yeso como acabados más duraderos. Esta solución, tradicional en zonas donde escasean otro tipo de recursos, ha caracterizado la imagen de conjuntos arquitectónicos de reconocido valor etnográfico, como ocurre en la zona de Tierra de Campos y aledaños, en la confluencia de las provincias de León, Palencia, Valladolid y Zamora. Debido a su limitada durabilidad, los ciclos de renovación eran anuales, y por tanto hoy en día debemos recurrir a soluciones estabilizadas algo más duraderas. En este sentido y según las investigaciones realizadas por el autor de este artículo, en su trabajo de tesis doctoral, la utilización de productos naturales o sintéticos como estabilizantes, diluidos en el agua de amasado del barro, ha resultado la solución más eficaz (7), siendo los productos comerciales con base de resinas sintéticas los que mejores prestaciones han ofrecido en los ensayos realizados ${ }^{9}$. Si no se utilizan estas soluciones mejoradas, por lo general el revestimiento de barro necesitará de una imprimación superficial con otros productos, como se ha detallado en el apartado anterior (Figuras 6 a 9).

Lo más frecuente es utilizar conglomerantes tradicionales como yeso, cal (aérea o hidráulica) o cemento, o toda una suerte de compuestos basados en estos productos, combinados con algunos de los aglutinantes, impermeabilizantes, ligantes, etc. mencionados al referirnos a las pinturas y con la propia tierra como árido para la fabricación del mortero.

Los revocos de cemento no son los más adecuados para revestir fábricas de este tipo, dada la excesiva diferencia de rigidez con

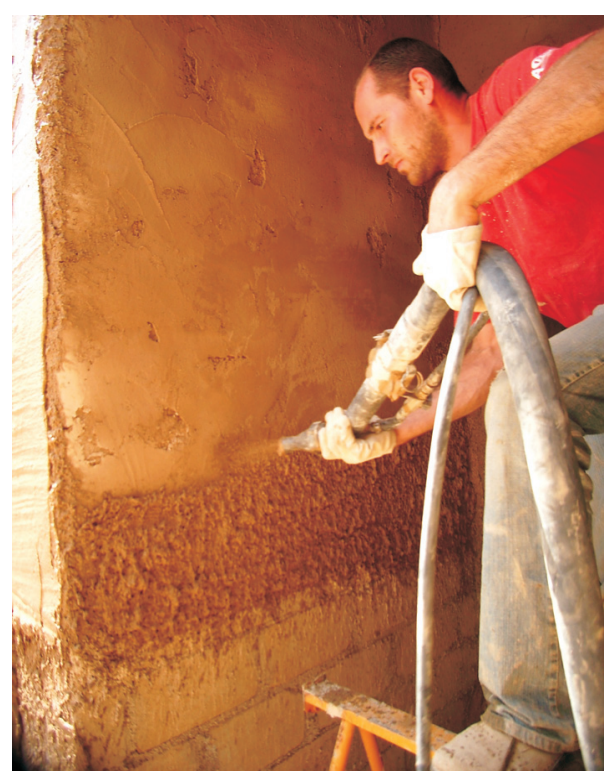

los muros (que origina la aparición de fisuras y desprendimiento del mismo) y excesiva impermeabilidad, no permitiendo el secado cuando estos se humedecen a través de las fisuras del propio mortero, como demuestran numerosos testimonios sobre intervenciones realizadas en otros países en conjuntos históricos ${ }^{10}$ o un simple paseo por muchos de los pueblos de la península, donde la aplicación indiscriminada de enfoscados o morteros monocapa industriales ofrece resultados devastadores (Figura 10).

No obstante, durante muchos años, los revocos y pinturas de cemento han sido defendidos como la mejor alternativa incluso desde las instituciones, como el Departamento de Agricultura de Estados Unidos (8, 9), donde la adherencia con el muro de tierra se solventa con la inclusión de mallas de alambre (simple torsión o tela de gallinero) ${ }^{11}$ clavadas sobre el muro previo a la aplicación del mortero (Figura 11).

Esta solución es bastante común hoy en día, minimizando el riesgo de aparición de fisuras y el desprendimiento, mejorando en cierta medida la adherencia, pero empeorando la situación del muro frente a la filtración de agua por otras vías, por lo que sólo parece admisible cuando existen garantías de que el muro de tierra ha "secado" convenientemente tras su construcción (o su reparación, aireación, etc. en caso de una rehabilitación) y queda bien confinado mediante otros elementos constructivos (zócalos, aleros).

Mucho más adecuados y mundialmente aceptados son los revocos de cal. Los morteros de cal empleados normalmente constan de 1 parte de cal y de 2 a 4 de arena, siendo esta proporción corriente en casi todo el mundo. Tradicionalmente se aconsejaba la adición de pelo de animales y caseína para disminuir la fisuración y la absorción de agua y aumentar la difusividad del vapor, al igual que para las pinturas (Figuras 12 y 13).

El principal problema que plantean los revocos es su adherencia al soporte, especialmente en paramentos con poca rugosidad (tapias bien compactadas y BTC). Tradicionalmente se recurría a distintas alternativas, como el picoteado de la fábrica ${ }^{12}$ o la inserción de elementos de agarre como cascotes o clavos. En muchas zonas es frecuente la introducción de ladrillos huecos adosados al tapial alternativamente que hacen de Ilave de unión permitiendo cierta penetración del mortero: soluciones todas ellas perfectamente aplicables hoy en día en trabajos de restauración o sobre fábricas modernas.

En cualquier caso, la mejor forma de garantizar el buen funcionamiento del revoco es 


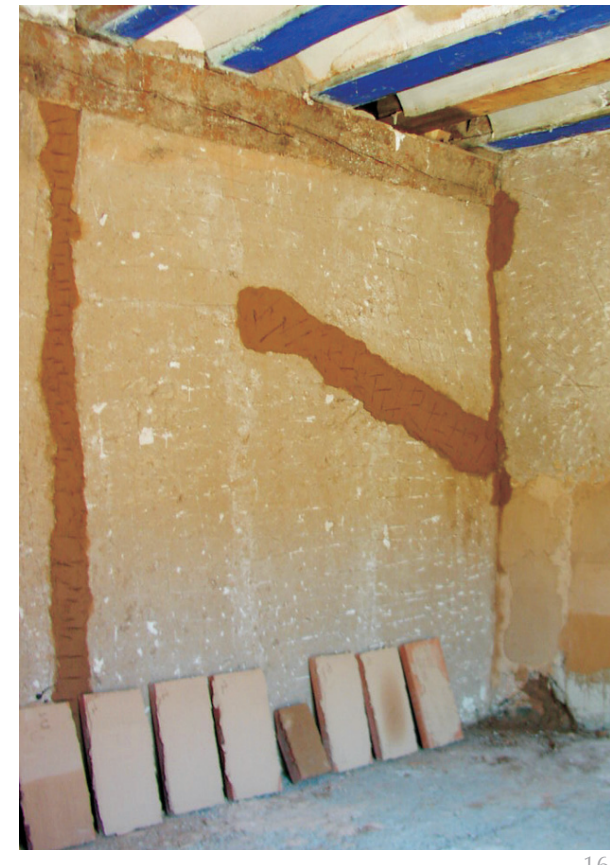

aplicarlo preferiblemente en varias capas (dos como mínimo) con un espesor no superior a $20 \mathrm{~mm}$. Para la aplicación del revoco es necesario tener en cuenta las mismas consideraciones a la hora de preparar el soporte que para cualquier otro tipo de fábrica de albañilería, es decir: que el paramento debe estar seco y limpio de material que pueda desprenderse y, previamente a la aplicación, se humedecerá ligeramente (o se dará una imprimación con una lechada de cal, o de material conglomerante similar al del revoco, que penetre unos milímetros en el soporte).

\subsubsection{Revestimientos Interiores}

Para el revestimiento de tabiques y caras internas de los muros se puede utilizar el mismo tipo de revocos en función del grado de exposición (según que se trate de cuartos húmedos o no). El revestimiento de barro es igualmente adecuado, aunque tradicionalmente iba acompañado de un enlucido de yeso o encalado final para dar mayor luminosidad. En otras culturas la utilización de barros muy finos como elementos de acabado interior, con fines decorativos, acompañados de una gran variedad de pigmentos naturales, ha caracterizado buena parte de la arquitectura vernácula.

Por otro lado, la arcilla (de modo similar al yeso) tiene algunas propiedades intrínsecas muy especiales para acondicionar un ambiente habitable. Es una superficie que transpira regulando tanto humedad como temperatura. Absorbe humedad haciendo más confortables los espacios en tiempo húmedo y cuando el ambiente se vuelve más seco desprende humedad hacia la habitación mejorando la calidad del aire.

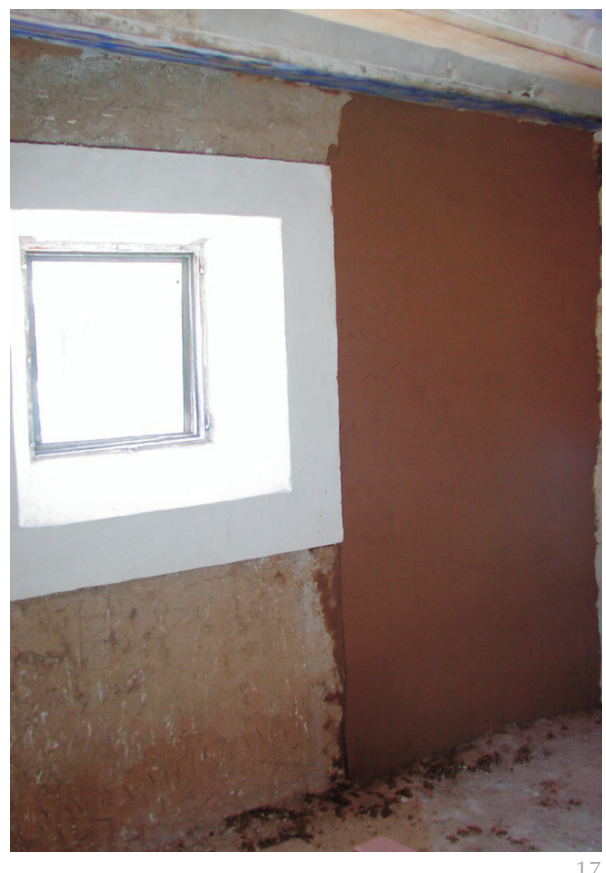

Asimismo esto supone una alternativa para la reutilización y reciclaje de la tierra como material de construcción procedente de las numerosas construcciones en ruina existentes hoy en día.

Como experiencia personal, este sistema se ha utilizado en la rehabilitación de una vivienda en la provincia de Soria, en la que todo el material de relleno de bajo cubierta, entrepisos y adobes procedentes de particiones interiores, se reutilizó, tamizado con cedazos de $2 \mathrm{~mm}$ de luz de modo que se obtuviera una tierra muy fina, que junto con distintas dosificaciones de arena y fibras permitiera obtener un revestimiento de aspecto natural, de fácil aplicación y reutilizable, con una textura y resistencia superficial adecuada.

\section{Tabla 1}

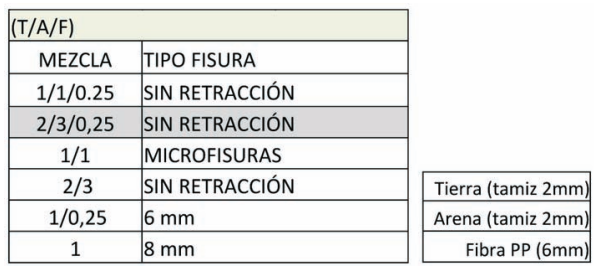

La tierra resultante tras el cribado es idónea, al contener una pequeña cantidad de "pajillas" de entre 6 y $12 \mathrm{~mm}$ de longitud, que pasan a través del tamiz; no obstante, su elevado contenido en arcilla hace que aun presente fisuración por retracción si se aplica en estado natural. Como opción se utilizaron adiciones de arena (con el mismo tamiz) y fibra de polipropileno.

Los mejores resultados son los proporcionados por una combinación de los tres ma-
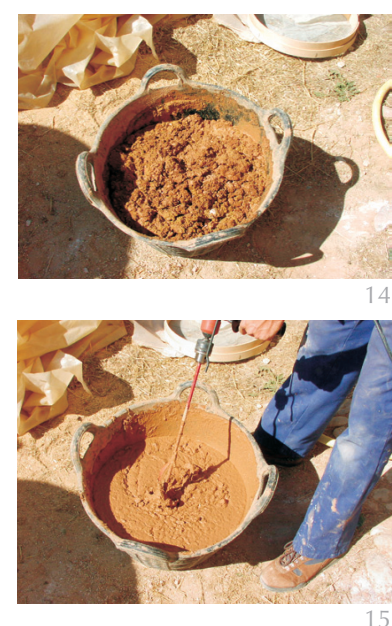

14-17. Aplicación de revestimiento de barro sobre capa base de trullado en paramentos interiores de adobe y mampostería. Rehabilitación de vivienda unifamiliar en Villasayas (Soria). Fotos 14 y 15. Preparación de la mezcla. Foto 16. Muestras con distintas dosificaciones y repello de la capa base con material de demolición reamasado, previo a la capa de terminación. Julio 2004

${ }^{9}$ En este caso concreto se utilizó SIKAlatex, Una emulsión acuosa de estireno-butadieno (SBR) en forma de líquido lechoso.

10 Minke (1996) menciona el caso de una de las viviendas más antiguas construidas con tapia en Meldorf (Alemania, 1795) donde las reparaciones realizadas en 1992 pusieron de manifiesto la erosión producida en el interior de los muros por el agua filtrada a través de las fisuras del revestimiento de cemento aplicado algunas décadas antes. O la iglesia de San Francisco en Ranchos de Taos, construida con adobe y embarrada en 1815, recubierta con un mortero de cemento en la década de 1960, que tuvo que retirarse once años después.

${ }^{11}$ Generalmente de acero galvanizado. Más recientemente se han empezado a utilizar mallas de fibra de vidrio, pvc o polipropileno.

${ }^{12}$ El soporte se puede humedecer hasta que esté suficientemente blando para realizar incisiones formando dibujos de líneas entrecruzadas (preferentemente a $45^{\circ}$ respecto de la horizontal). 
18-21. Diferentes muestras de productos para revestimientos de barro en la feria internacional de construcción con tierra Lehm 2008. Koblenz (Alemania)
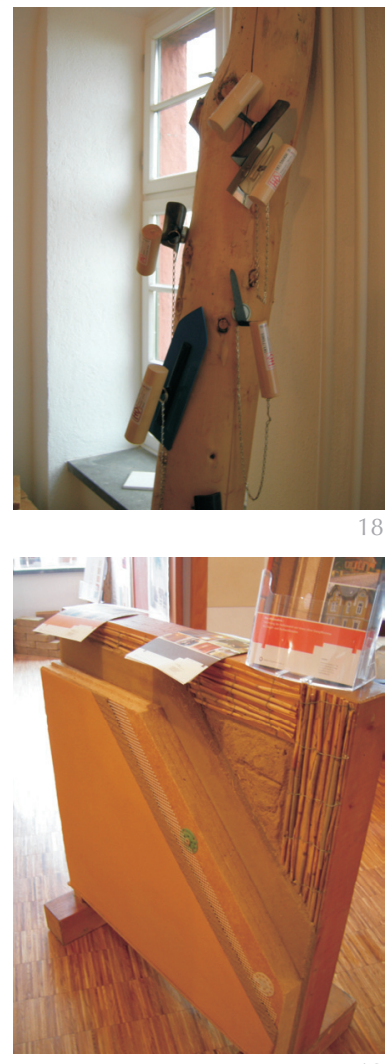

${ }^{13} \mathrm{http} / / / \mathrm{www}$.claytec.de/produkte/baustoffe.html

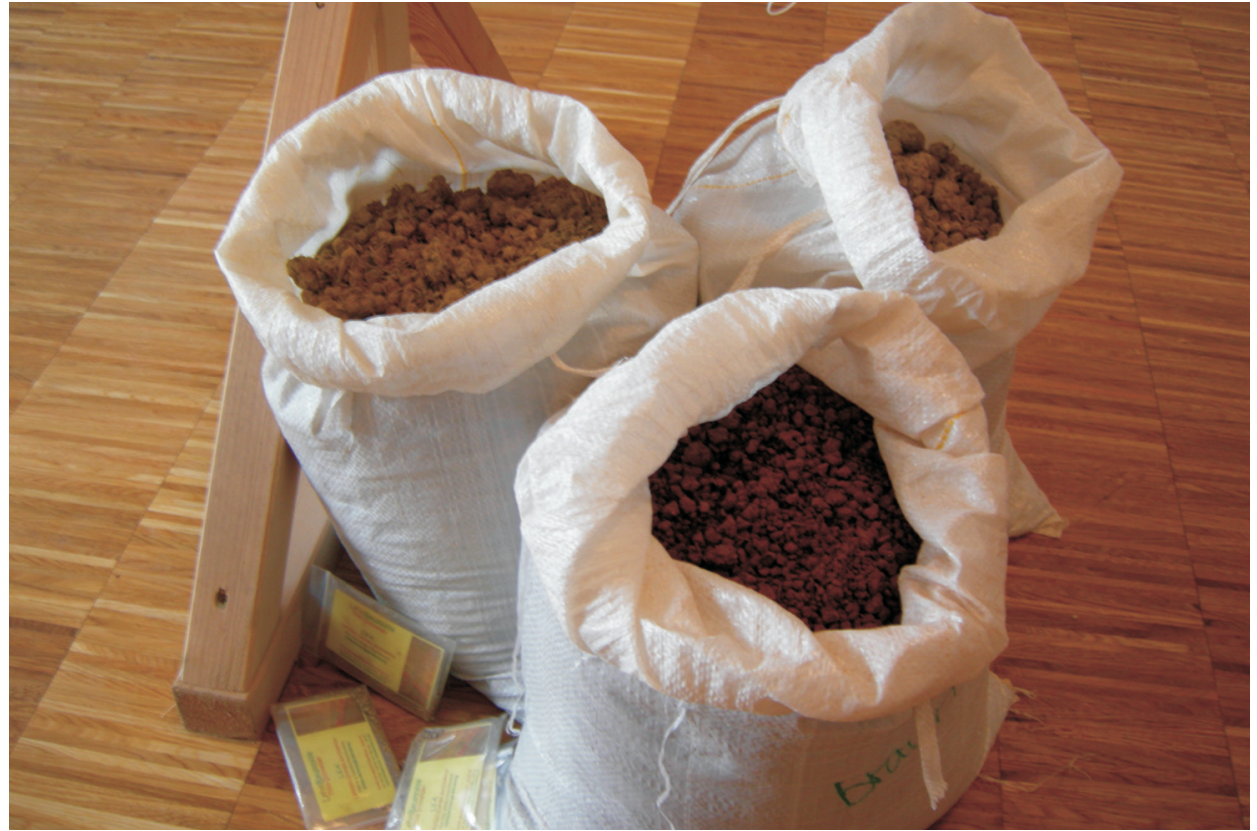

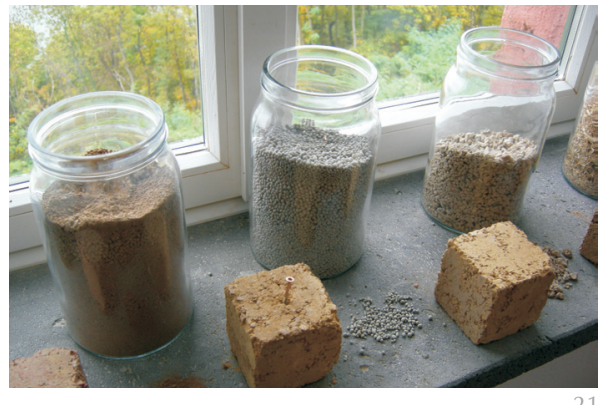

teriales en las proporciones indicadas en la (tabla 1), con los que se obtiene un revestimiento liso, sin fisuras y de buena resistencia superficial. Los materiales se mezclan en seco y el amasado se realiza con una batidora eléctrica de pintor. El revestimiento se aplica sobre la capa de embarrado existente, muy agrietada pero bien adherida al soporte y repellada con el propio material de demolición reamasado en aquellas zonas donde aparecen desprendimientos (Figuras 14 a 17)

Este tipo de técnicas tienen una relación estrecha con las mezclas utilizadas en la arquitectura tradicional. Se suprime la cal utilizada antiguamente y se aumenta la cantidad y la calidad de la arena, buscando la proporción adecuada.

Hoy en día es posible encontrar soluciones comerciales similares, aunque por lo general de empresas extranjeras, con productos de tipo mineral ensacados y preparados para su utilización por simple amasado con agua. Estos productos tienen como base tierras seleccionadas y encuentran una difusión importante en mercados del centro de Europa en el sector de la bioconstrucción ${ }^{13}$ (Figuras 18 a 21). Las exigentes demandas de este tipo mercado (respecto a la composición de estos productos supuestamente "naturales") ha propiciado la elaboración de una normativa en Alemania sobre los requisitos que deben cumplir para poder comercializarse como tales (10).

\section{CALICASTRADO}

Si existe un tipo de revestimiento característico en la península es el denominado "calicastrado" (o costra de cal), muy habitual en la construcción militar de la época almohade y difundido especialmente en la construcción popular de la Submeseta Sur y Levante. Consiste esta técnica en proporcionar el revestimiento durante el mismo proceso de apisonado del tapial, obteniendo así una tapia reforzada en las caras o acerada, donde estos materiales de refuerzo se disponen junto a las caras del tapial antes del apisonado de cada tongada, quedando así íntimamente ligado el revoco a la masa del muro. Este careado suele hacerse con mezcla de cal y arena que recibe el nombre de "malhecho" (según testimonios orales de albañiles de la provincia de Albacete) y una vez endurecido se le denomina "costra", refiriéndose al muro como "tapia con costra" o "calicostrada". La mezcla (una parte de cal por tres de arena normalmente) se pone "en el mismo estado de humedad que la tierra; esta mezcla se extiende a lo largo de los tableros y arrimada a ellos" (11).

Hoy en día esta solución sigue siendo un procedimiento adecuado en los trabajos de reconstrucción de muros de gran espesor (11), que combinado con las técnicas y herramientas de construcción contemporáneas, permite la ejecución de muros de gran calidad (Figuras 22 a 24). 

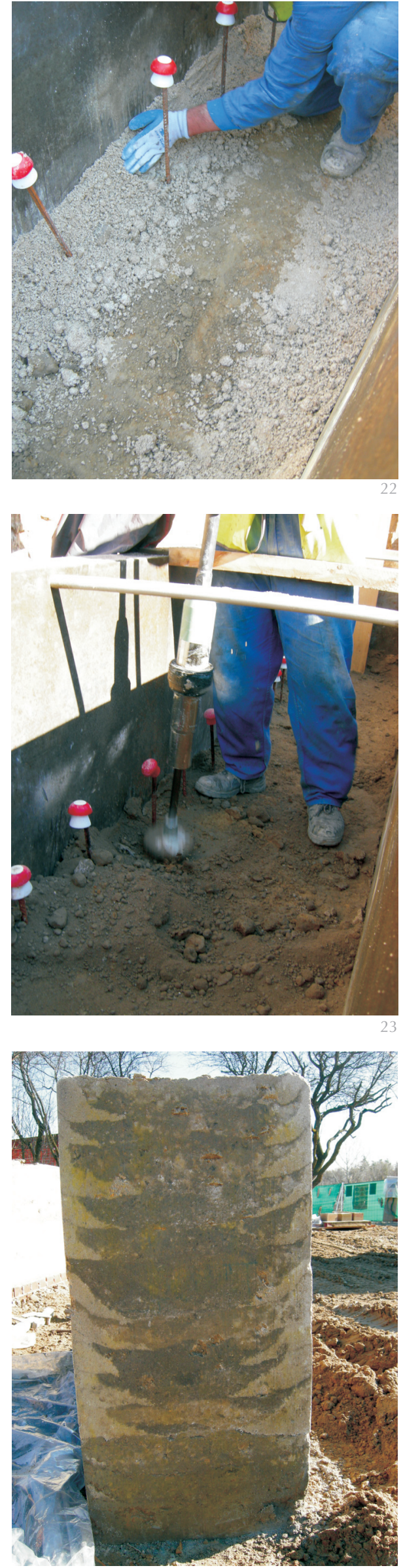

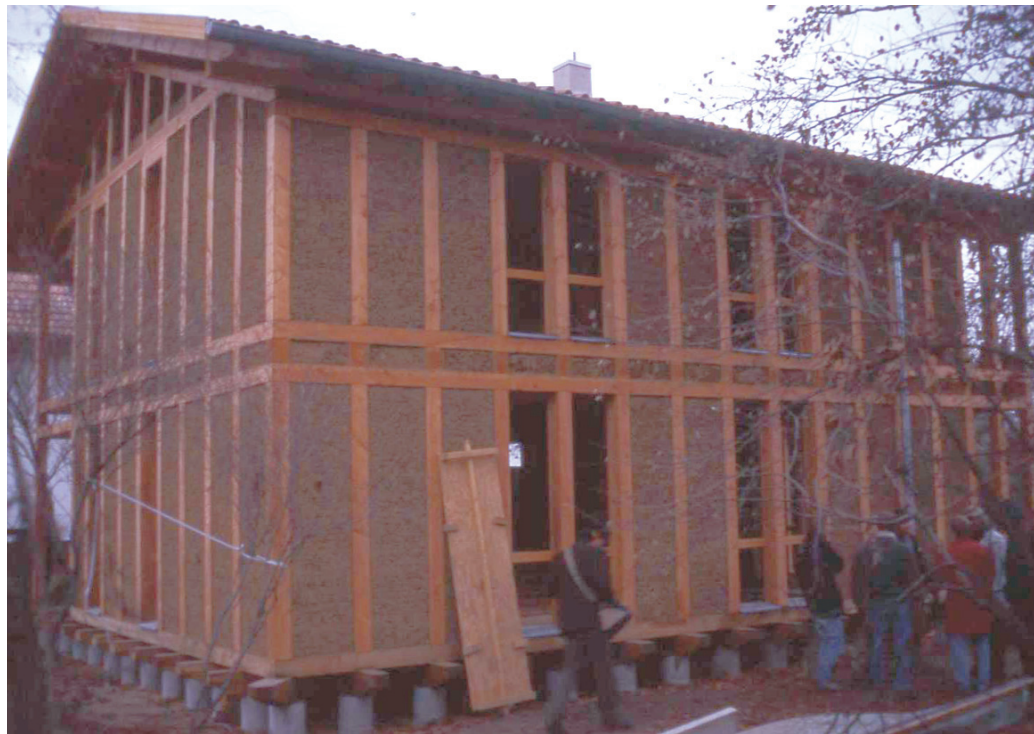

\section{REVESTIMIENTOS Y APLACADOS CON OTROS MATERIALES}

Como alternativa para los revestimientos de cerramientos exteriores hay que mencionar la posibilidad de realizar el revoco sobre una capa intermedia de aislamiento, que se coloca por el exterior del muro. Esta es una solución habitual en regiones como el sur de los EEUU, donde el sistema constructivo habitual de estas viviendas está compuesto por muros de carga de adobe, con un espesor mínimo de 25 o $35 \mathrm{~cm}^{14}$, trasdosados exteriormente con aislamiento rígido de poliestireno extruido (4 cm generalmente) y revestimiento exterior continuo de cemento coloreado. El aislamiento se fija mecánicamente al muro. Este tipo de soluciones resultan muy adecuadas para aprovechar la inercia térmica de los muros, lo que puede combinarse con el calentamiento pasivo de los mismos o los sistemas de calefacción/refrigeración de baja temperatura (paramentos radiantes).

Finalmente, la protección del muro de tierra mediante piezas de otro material no es muy habitual, dada la dificultad de soportar esfuerzos de corte producidos por el peso de las propias piezas, si estas se reciben directamente en el muro con morteros, o por la estructura auxiliar (generalmente de madera) necesaria para colgarlas. Lo habitual es encontrar este tipo de soluciones en muros de entramado y relleno de tierra, donde los elementos del entramado suelen servir de sujeción al revestimiento exterior, generalmente de tablas (latas, chillas, ripias, etc). Muy característico en la arquitectura popular de comarcas como La Vera (Cáceres), La Alberca (Salamanca) o la montaña Palentina y Leonesa. Las viviendas centroeuropeas construidas con entramados de madera pesados y rellenos de tierra propician

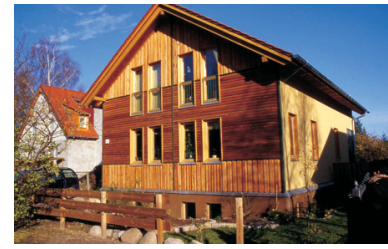

22-24. Ejecución de muro de tierra construcción del aula de Naturaleza en Pozuelo de Alarcón (Madrid) Foto 24. Muro de muestra realizado durante la obra. Enero 2008

25 y 26. Vivienda con cerramiento $y$ estructura de entramado de madera elleno con tierra aligerada (con virutas de madera) y acabado exterior con entablado de madera. Vivienda unifamiliar. Berlín-Mahlsdorf (Alemania) 1998/2001

${ }^{14}$ Las dimensiones medias de los adobes son de $4 \times 10 \times 14$ pulgadas $(10 \times 25 \times 35 \mathrm{~cm})$. El espesor del muro depende de su colocación en función de la altura. apisonada con costra de cal en la 
una situación adecuada para la utilización de este tipo de revestimientos (Figuras 25 y 26), por lo que es frecuente encontrar soluciones basadas en esta técnica en países como Alemania, Suiza o Austria.

\section{CONCLUSIONES}

Como conclusión, no existe un tratamiento ideal para el revestimiento de los muros de tierra, siendo el abanico de posibilidades muy grande. En la mayoría de los casos resultan más relevantes las condiciones y forma de aplicación de los revestimientos, una vez comprobada su compatibilidad con el soporte, que la composición del material.

En el caso de las sustancias utilizadas para impregnaciones e imprimaciones deben reunir las siguientes características, que deberíamos procurar confirmar con la documentación técnica de cada producto y no con la documentación comercial.

- Capacidad para mantener la porosidad del soporte y permitir el paso del vapor de agua

- Capacidad de admisión de nuevos tratamientos
- Inalterabilidad de color

En el caso de los productos utilizados como aditivos para morteros de barro, las resinas sintéticas (de Estireno Butadieno, SBS) diluidas en el agua de amasado y en la lechada de humedecimiento previo del muro, han demostrado un buen comportamiento en las experiencias presentadas. La diversidad de productos y composiciones dentro de esta misma familia y la falta de información específica sobre los mismos en la mayoría de los trabajos no permiten sacar conclusiones concretas aplicables a otros productos similares existentes en el mercado, por lo que es necesario profundizar en las investigaciones sobre la aplicación de este tipo de productos. La forma de aplicación es asimismo determinante y en consecuencia cada producto debería ser probado previamente sobre el material específico (tipo de tierra) en el que se va a aplicar comprobando sus efectos.

Esta solución debe estar además sometida a renovaciones periódicas, cuya periodicidad es difícil de determinar a priori, ya que estará en función del grado de exposición del paramento y del grado de dilución del producto utilizado.

\section{BIBLIOGRAFÍA}

(1) Romero Taylor, G.: "An evaluation of the New Mexico state monuments test walls at Fort Selden". Adobe 90. preprints. 6th International conference on the conservation of earthen architecture (1990), pp. 383-389. Getty Conservation Institute.Los Angeles, Las Cruces, Nex Mexico.

(2) Chiari, G.: "Ethyl Silicate treatments and Humidity". Terra 93 (1993), pp. 422-425.

(3) Beas Guerrero de Luna, M. I.: "Consolidation of traditional plasters: A laboratory research". Terra 93. Comunicaçôes. $7^{a}$ conferencia internacional sobre o estudo e conservaçao da arquitetura de terra (1993), pp. 410-416. Direcçâo Geral dos Edifiçios e Monumentos Nacionais, Lisboa.

(4) Facey, W. Back to earth. Adobe building in Saudi Arabia. pp. 145-146, Al-Turath, Riyadh, 1997.

(5) Torres Soria, P.; Cedillo Álvarez, L.: "La goma de nopal: una aportación para la conservación de arquitectura de tierra seca protegida por la techumbre"(2003). NRPC - INAH. http://www.conservacionyrestauracion.inah.gob.mx/html/correo_restaurador/html/CORE0411.html

(6) Minke, G. Lehmbau - Handbuch. Ökobuch. Freiburg, 1994: Earth Construction Handbook. WITpress, Southampton, 2000 (traducción del anterior) y Manual de construcción en tierra, pp. 114-116, Fin de Siglo, Uruguay, 2008.

(7) Castilla, F.: "Estabilización de morteros de barro para la protección de muros de tierra (mejora de la durabilidad)". Actas de las I Jornadas de Investigación en Construcción (2005), pp. 717-737. Instituto de Ciencias de la Construcción "Eduardo Torroja", Madrid.

(8) Merill, A. F.: Casas de tierra apisonada (The rammed-earth house). traducido por Jose Luis Moia, Universidad Nacional de Buenos Aires. Ed Windsor, Buenos Aires, 1948.

(9) Hunter, T.: "Building with adobe and stabilized- earth blocks". United States Department of Agriculture. Leaflet 535 (1978). Washington.

(10) Minke, G.; Ziegler, K.: "The requirements for interior clay plasters". Actas del congreso "Lehm 2008" (2008), pp. 23-31. Organizado por Dachverband Lehm e.V, Koblenz.

(11) Castilla, F.: "La técnica del Tapial en la Construcción Tradicional de la Provincia de Albacete". Revista Zahora. Volumen no 45 (2006), p. 37. Diputación provincial de Albacete. 